\title{
Surface Chemistry of Functionalized Carbon Nanotubes
}

\author{
Z.P. Luo, ${ }^{a}$ L. Carson, ${ }^{b}$ A. Oki ${ }^{c}$, L. Adams, ${ }^{c}$ N. Soboyejo, ${ }^{c}$ E.G.C. Regisford ${ }^{d}$ \\ ${ }^{a}$ Microscopy and Imaging Center, Texas A\&M University, College Station, TX 77843-2257 \\ ${ }^{\mathrm{b}}$ Cooperative Agricultural Research Center, Prairie View A\&M University, Prairie View, TX 77446 \\ ${ }^{c}$ Department of Chemistry, Prairie View A\&M University, Prairie View, TX 77446 \\ ${ }^{\mathrm{d}}$ Department of Biology, Prairie View A\&M University, Prairie View, TX 77446
}

Carbon nanotubes (CNTs) have been well recognized as a promising material due to their extraordinary mechanical, thermal and electrical properties for diverse applications. However, their behavior of hydrophobicity and chemical inertness, which cause tangling or poor dispersion, limits their commercial applications. Towards this technical issue, it has been found that the functionalization of CNTs by surface modification using chemical reactants [1-4] is an effective way to improve the dispersion degrees [5]. In order to have a better understanding of the mechanism, a characterization of the surface chemistry of various functionalized CNTs is performed in this work.

Four different kinds of materials were used: pure CNT (PV40), CNT-COOH (PV22), CNT-COCl (PV33), and CNT-F (PV49). The first sample of pure CNT was used for comparison. The second sample, CNT-COOH, was derived by reacting CNTs with mixed sulfuric and nitric acids. The third sample, CNT-COCl, was prepared by further reacting $\mathrm{CNT}-\mathrm{COOH}$ with thionyl chloride $\mathrm{SOCl}_{2}$. The last sample, CNT-F, was fluorinated. The CNT samples were deposited on $\mathrm{Cu}$ grids coated with holly Si-O-C support film, and observed in a FEI Tecnai $\mathrm{G}^{2}$ F20 at $200 \mathrm{kV}$, equipped with a Fischione ultra-high resolution STEM HAADF detector, Oxford instruments EDS detector, and a post-column Gatan Image Filter (GIF).

The appearance of the functionalized CNTs is shown in Fig. 1. The unfunctionalized CNTs exhibit almost clean surface, as shown in Fig. 1(a). An enlargement is inserted. However, the samples CNT$\mathrm{COOH}, \mathrm{CNT}-\mathrm{COCl}$, and CNT-F present evident reactants on their surfaces, as shown in Figs. 1b - d respectively. In the CNT-COOH sample (b), the CNT with three layers is coated with about half surface areas. The EDS analysis reveals evident N, O and S peaks. In the CNT-COCl sample (c), the black particles are identified as Co nanoparticles, and the reactants contain $\mathrm{N}, \mathrm{O}, \mathrm{Cl}$, and $\mathrm{Cl}$ from EDS. In the CNT-F sample in (d), the CNTs are almost entirely coated with thin layers, which exhibit $\mathrm{N}, \mathrm{O}$ and $\mathrm{F}$ from EDS.

A STEM Z-contrast image of the sample CNT-COOH is shown in Fig. 2(a). It is evident that the CNTs are heavily coated. The bright nanoparticles are metal Co that gives much higher scattering contrast. A line scan is performed crossing a single CNT as indicated. The framed area is used for reference to correct any drifts during the data acquisition. The results of the normalized counts of $\mathrm{C}$, $\mathrm{N}, \mathrm{O}$ and $\mathrm{S}$ (all K lines) are shown in Fig. 2(b). At the CNT position, the EDS counts are higher, which indicates that they come from the CNT rather than spurious signals. The coating on the CNTs provides excellent bonding to prepare CNT/chitosan nanocomposites [6,7].

References

[1] K.A. Wepasnick, B.A. Smith, J.L. Bitter et al. Analy. Bioanly. Chem. 396 (2010) 1003.

[2] L. Adams, A. Oki, T. Grady, et al. Physica E41 (2009) 723. 
[3] A. Oki, L. Adams, Z.P. Luo, et al. J. Phys. Chem. Solids 69 (2008) 1194.

[4] A. Oki, L. Adams, V. Khabashesku, et al. Mater. Lett. 62 (2008) 918.

[5] Z.P. Luo et al. J. Microsc. 225 (2007) 118; Polymer 49 (2008) 1841; Mater. Lett. 62 (2008) 3493.

[6] L. Carson, C. Kelly-Brown, M. Stewart, et al. Mater. Lett. 63 (2009) 617.

[7] The authors thank NIH-NIAMSD Grant \# ARI49172, NIH-NIGMS RISE Grant \#1 R25 GM078361-01, the Welch Foundation, the US Air Force Research Laboratory, and TAMU VPR Office for supporting this work.
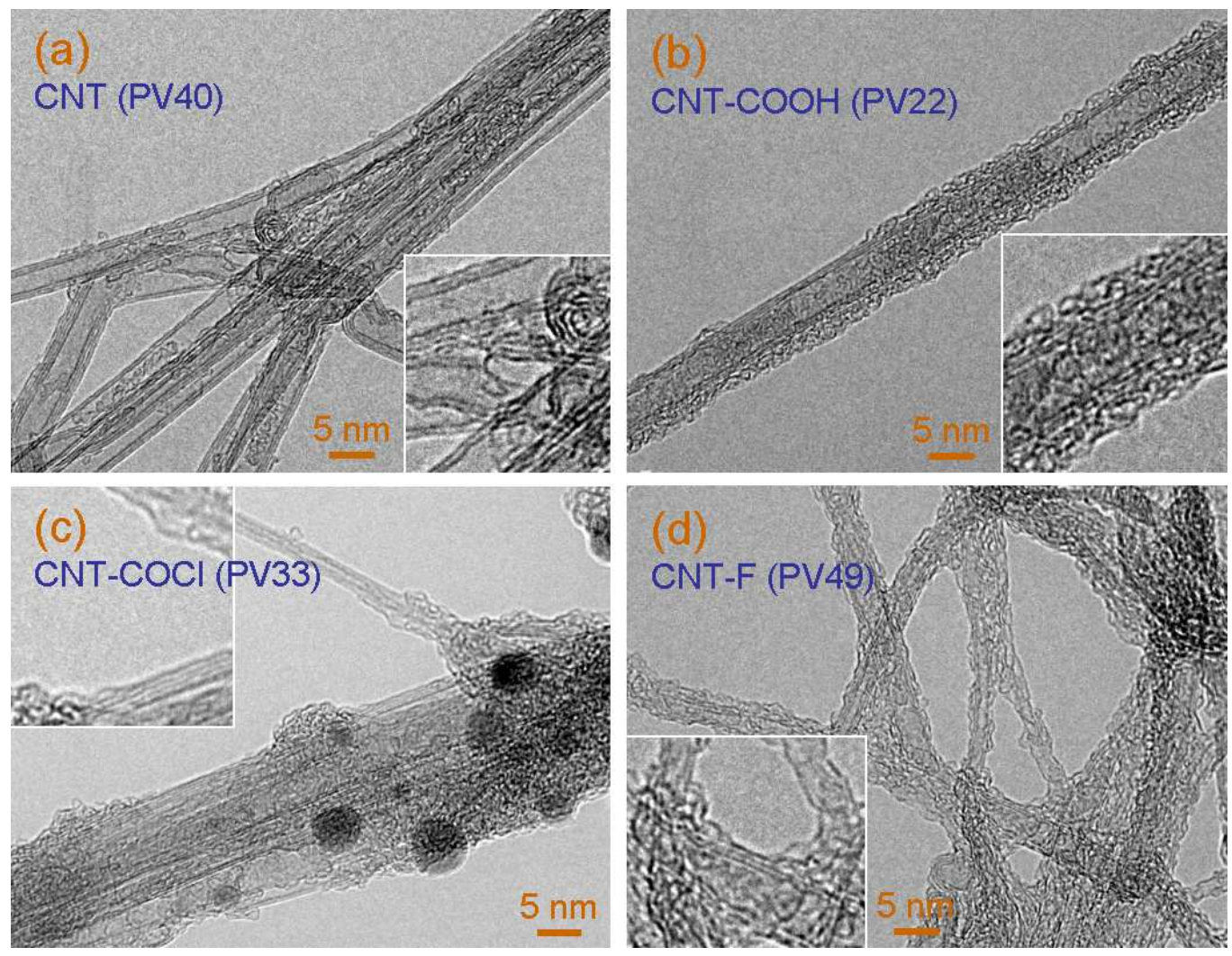

Fig. 1. Zero-loss TEM images of four samples.
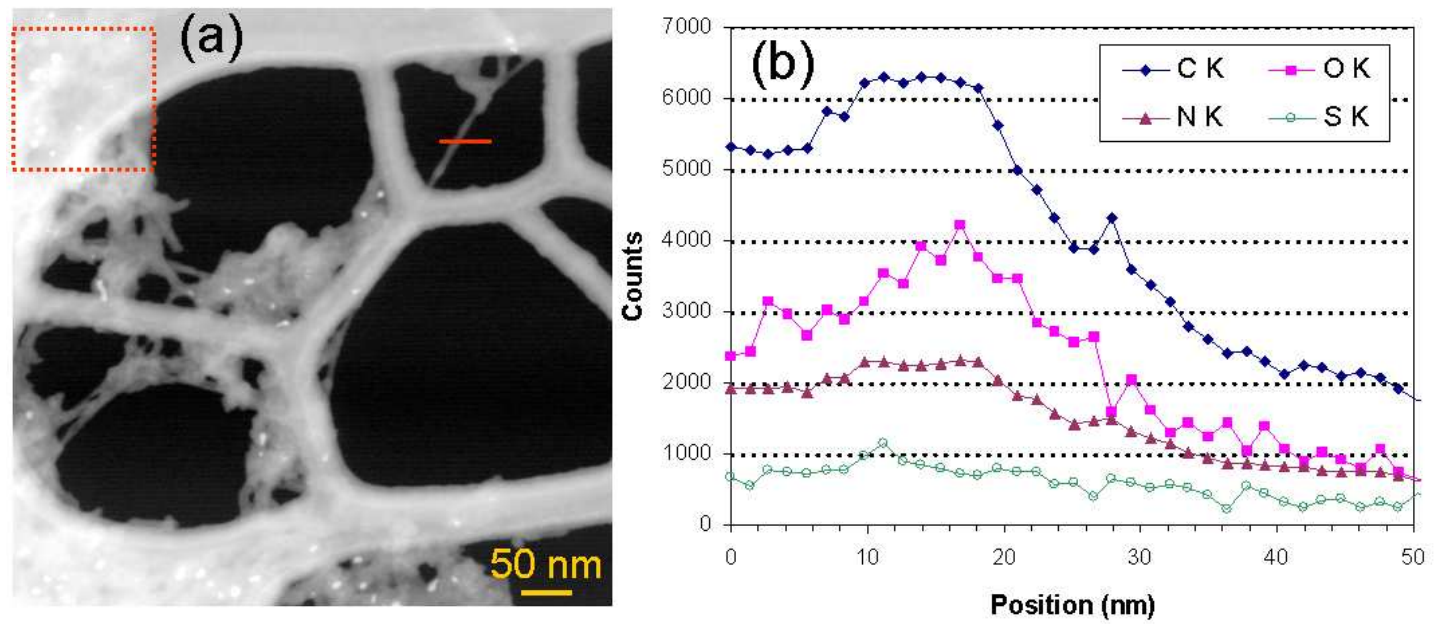

Fig. 2. (a) STEM image of CNT-COOH sample; (b) EDS line scan profiles. 